\title{
Design of a Surface Plasmon Resonance Sensor Based on Grating Connection
}

\author{
Jun ZHU ${ }^{1 *}$, Liuli QIN ${ }^{2}$, Shuxiang SONG ${ }^{1}$, Junwen $\mathrm{ZHONG}^{1}$, and Siyuan $\mathrm{LIN}^{1}$ \\ ${ }^{1}$ College of Electronic Engineering, Guangxi Normal University, Guilin, 541004, China \\ ${ }^{2}$ College of Mathematics and Statistics, Guangxi Normal University, Guilin, 541004, China \\ *Corresponding author: Jun ZHUＥ-mail: zhujun1985@gxnu.edu.cn
}

\begin{abstract}
Nowdays, the study of measurement of the biological field focuses on the research of improving surface plasmon resonance (SPR) in the fields of integration and detection sensitivity. We designed a kind of grating connected surface plasmon resonance sensor. Theoretically, we analyzed the wave vector and the effective refractive index relations with the diffraction grating structure. Then we obtained the nanoparticles enhancement SPR structure with a resolution 10 times higher than that of traditional SPR sensors. Also, we used the finite-difference time-domain (FDTD) analysis and simulation which showed that it was obvious with coupling effect by the nanoparticles enhancement SPR structure that the reflectance spectral bandwidth results validated the structure significantly which improved the sensitivity. Experimental results showed that the dynamic response of the designed sensor reached $10^{-6}$ RIU (refractive index unit). This study has the certain significance to long-distance and special sensing applications.
\end{abstract}

Keywords: Surface plasmon resonance, waveguide grating, mode coupling, sensitivity, resolution

Citation: Jun ZHU, Liuli QIN, Shuxiang SONG, Junwen ZHONG, and Siyuan LIN, "Design of a Surface Plasmon Resonance Sensor Based on Grating Connection," Photonic Sensors, 2015, 5(2): 159-165.

\section{Introduction}

Surface plasmon (SP) is the local oscillation of free electrons on a metal surface. When the transversal magnetic (TM) incident fading wave phase velocity matches with the phase velocity of SPs, the surface plasmon resonance (SPR) phenomenon occurs. Moreover, all incident photon energy will shift to SPs [1-3]. Compared with the traditional detection method, the current SPR sensors are mostly based on the Kretschmann device, following the principle of the attenuated total reflection (ATR) prism coupling method to stimulate the SPs, thus resulting in a better detection limit [4, 5]. In the device, the determination of real-time information related to the interaction among molecules on the surface of the sensor is possible by monitoring the SPR reflection spectrum of the tilted angle offset [6,7]. However, the measurement of molecules using a highly complex and precise measurement system, such as the Kretschmann device, still has some drawbacks [8]. Therefore, in the case of not using the ATR prism coupler, improving the ability of the SPR technique for studying molecular interactions is necessary. In a recent study, the application of a 2-dimension (2D)

Received: 1 February 2015 / Revised version: 13 March 2015

(C) The Author(s) 2015.This article is published with open access at Springerlink.com DOI: 10.1007/s13320-015-0244-1

Article type: Regular 
diffraction grating structure to molecular detection conducted by Cunningham et al. achieved a resolution of 0.1 protein layer thickness [9]. Kunz et $a l$. integrated the wavelength tunable semiconductor lasers and the phase-locked amplifier to detect a considerable number of media or small molecular mass range, which was equivalent to $0.3 \mathrm{pg} / \mathrm{mm}^{2}$ [10]. The detection limit refractive index unit (RIU) reached $10^{-6}[11]$. The detection limit of the sensing system is based on two important factors: the developed sensor sensitivity and the resolution of the developed instrument in the sensor $[12,13]$. The most important differences between the two sensors are their diffraction grating structure and their method of testing application [14]. Cunningham et al. showed that the spectroscopic detection resolution was $0.01 \mathrm{~nm}$, which was approximately 50 times worse than that reported by Kunz et al. Brolo et al. developed a sensor based on the plasmon effect, which was a result of incident light transmission in periodic nanostructures $[15,16]$. Although this kind of sensor has achieved the high sensitivity, its wavelength resolution remains poor because its transmission wavelength spectrum is wide [17]. However, the surface plasmon Bragg grating sensor is still based on the Kretschmann device despite its defects. Dostaleket et al. [18] also reported a new kind of SPR sensor based on the grating structure array to detect 216 channels at the same time. However, the reflection resonance spectrum is still wide [19]. Other researchers have proposed a new method to achieve a significant material detection limit of $9.4 \times 10^{-8}$ RIU that resulted in the formation of small subwavelength nanopore arrays on the metal film $[10,13]$. Based on this background, this study developed an SPR sensor based on the subwavelength grating waveguide structure. The waveguide structure provides the high detection sensitivity and high measurement resolution.

\section{Theory analysis and structure design}

\subsection{SPR theoretical analysis}

The vibration of the surface plasmon causes the metal/dielectric interface to produce a surface plasma wave with a field vector that is exponentially attenuated from the surface to two kinds of media. The plasma wave is a TM polarization wave, i.e., a shear wave, with an electric field vector that is perpendicular to the interface and magnetic field vectors that are parallel to the interface and perpendicular to the direction of propagation. We assume that the surface plasma wave angular frequency is $w_{\mathrm{ms}}$ and is located at the half infinite dielectric and metal interface wave vector, which is expressed as follows:

$$
\overline{k_{\mathrm{spw}}}=\frac{w_{\mathrm{ms}}}{c} \sqrt{\frac{\varepsilon_{m} \varepsilon_{a}}{\varepsilon_{m}+\varepsilon_{a}}}
$$

where $c$ is the speed of light in a vacuum, and $\varepsilon$ and $\mu$ are the metallic and dielectric permittivities, respectively. The ratio between the speeds of the electromagnetic wave in a vacuum, $c$, and the electromagnetic wave in the conductive homogeneous medium, $v$, is called the dielectric refractive index, $n$, which is expressed as follows:

$$
n=\frac{c}{v}=\sqrt{\varepsilon \mu} .
$$

Considering the light frequency, the dielectric refractive index is commonly nonmagnetic. If $\mu \approx 1$, then $n=\sqrt{\varepsilon}$, the light wave vector $k_{a}$ frequency is $\omega$, and the transmission by the dielectric is $k_{a}=\frac{w_{a}}{c} \sqrt{\varepsilon_{a}}=\frac{w_{a}}{c} n_{a}$. Therefore, for the resonance between the light and the surface plasma wave to occur, $k_{\mathrm{spw}}$ should be equal to $k_{a}$.

In the dielectric refractive index, the relationship between $\omega\left(k_{a}\right)$ and $\omega\left(k_{\mathrm{spw}}\right)$ is $\omega\left(k_{\mathrm{spw}}\right)<\omega\left(k_{a}\right)$, and no cross phenomenon is observed. Therefore, adopting the appropriate method to enable the intersection of $\omega\left(k_{a}\right)$ and $\omega\left(k_{\mathrm{spw}}\right)$ is necessary and is likely to shed light on the dielectric refractive index that directly 
stimulates the SPR. The use of the optical coupler can achieve this purpose in the practical application. Subsequently, the Kretschmann coupling model, e.g., the effects of the SPPs principle and the incident light as well as the cause of the resonance of SPPs, is further studied.

We assume that the interface wave vector of the surface plasma wave in the prism and metal surface is $k_{\text {spw01 }}$. The interface wave vectors in the metal and sample are expressed as follows:

$$
\begin{aligned}
& k_{\mathrm{spw} 01}=\frac{\omega_{p}}{c}\left(\frac{\varepsilon_{1}^{\prime} \varepsilon_{0}}{\varepsilon_{1}^{\prime}+\varepsilon_{0}}\right)^{\frac{1}{2}} \\
& k_{\mathrm{spw02}}=\frac{\omega_{p}}{c}\left(\frac{\varepsilon_{1}^{\prime} \varepsilon_{2}}{\varepsilon_{1}^{\prime}+\varepsilon_{2}}\right)^{\frac{1}{2}}
\end{aligned}
$$

where $\omega_{p}$ is the wave vectors angular frequency, $\varepsilon_{0}$ is the glass permittivities, and $\varepsilon_{1}^{\prime}$ and $\varepsilon_{2}$ are the metallic and dielectric permittivities, respectively. In the Kretschmann coupling model, if $\varepsilon_{0}>\varepsilon_{2}$, then $\omega\left(k_{\text {spw01 }}\right)>\omega\left(k_{\text {spw02 }}\right)$. Based on (3), (4), and (5), we can easily derive the dispersion curve by using the Kretschmann coupled model. As shown in Fig. 1, Curve A represents the surface plasma wave (SPW) dispersion relation at the metal and sample interface, and Curve B represents the SPW dispersion relation at the metal and prism interface. Slash I represents the SPW dispersion relation in the prism, which is a satisfied resonance condition. Slash II represents the SPW dispersion relation when $\theta=90^{\circ}$ in the prism, which is not a satisfied resonance condition. As shown in Fig. 1, by adjusting the incident angle, the diagonal lines representing Slash I and Curve A intersect at Point A. At that time, the SPW and the metal and sample interface have the same frequency and wave vector, which meet the condition of resonance. Therefore, the incident light can stimulate the SPR phenomenon and decrease the energy of the reflected light. Slash II and Curve B do not intersect, which means that the plane wave and the surface plasma of the metal and prism interface do not produce the resonance phenomenon.

Therefore, the factor that influences the incident light and the surface plasma, which cause the resonance phenomenon, only exists when the penetration depth of the disappearing wave is larger than the thickness of the metal film. If the thickness of the metal film is greater than the penetration depth of the disappearing wave, then the disappearing wave will not occur at the metal film and sample interface, and the SPR phenomenon will not yet occur. Therefore, in general, the thickness of the metal film is observed to be at the nanometer level. This finding fully supports our reason for adopting the $K$ model of ATR.

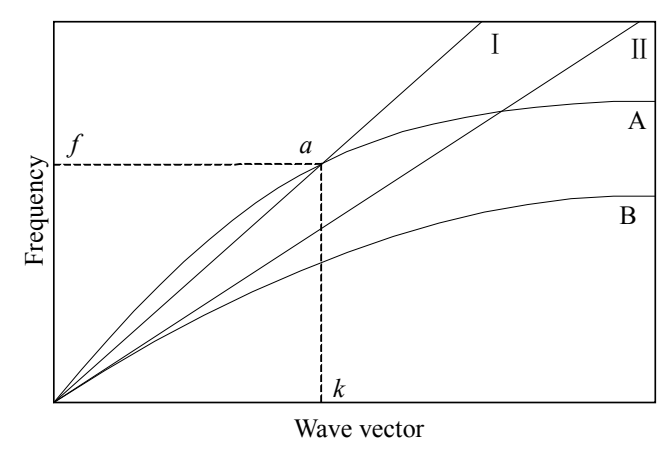

Fig. 1 Kretschmann coupled model of dispersion curves.

\subsection{Design structure and theoretical analysis}

The SPR sensor improves the sensitivity through the plasma effect. To stimulate the SPs at the metal/dielectric interface, the propagation constant of the incident white light of the TM mode should be equal to the SPs $k_{\mathrm{sp}}$ expressed as follows:

$$
k_{x}^{l}=k_{\mathrm{sp}} \cong \frac{2 \pi}{\lambda} \sqrt{\frac{\varepsilon_{m}^{\prime} \varepsilon_{b}}{\varepsilon_{m}^{\prime}+\varepsilon_{b}}}
$$

where $\lambda$ is the wavelength of the incident light, $\varepsilon_{m}^{\prime}$ is the real component of the metal/dielectric constant, and $\varepsilon_{b}$ is the dielectric medium of the constant. However, the propagation constant of the incident light on the surface of the parallel grating constant will change as follows:

$$
k_{x}^{l}=\frac{2 \pi}{\lambda} \sqrt{\varepsilon_{b}} \sin \theta+m \frac{2 \pi}{\Lambda}
$$

where $m$ is the order of diffraction, and $\Lambda$ is the 
cycle of the diffraction grating. In this design, gold and silver are ideal choices as the metal film in the visible light band. However, gold is more applicable to the plasma sensor because of its stability under the chemical reaction even if it has a wide spectrum.

For the gold film, if it stimulates SPs, a long wavelength is more suitable than a short wavelength. Therefore, we choose the $630-\mathrm{nm}$ resonant wavelength using (3) to obtain the propagation constant of SPs. Based on (4), when the plain white diffraction light $(\theta=0)$ is incident to the $m=1$ subwavelength grating, the new propagation constant can increase and match to the propagation constant of SPs under a particular wavelength matching, e.g., (3). The cycle of the diffraction grating can be determined. Therefore, the SPR phenomenon also occurs.

Figure 2(a) shows the structure of the SPR sensor with the subwavelength grating waveguide. The sensor consists of the buffer solution $\left(\varepsilon_{b}\right)$, molecular layer $\left(\varepsilon_{a}, d_{a}\right)$, metal layer $\left(\varepsilon_{m}, d_{m}\right)$, and dielectric waveguide layer $\left(\varepsilon_{w}, d_{w}\right)$. The dielectric waveguide layer is placed on the surface of the base $\left(\varepsilon_{s}\right)$, and its surface is sealed into the shape of a diffraction grating. $\varepsilon_{i}$ and $d_{i}$ represent the dielectric constant and the thickness of each layer, respectively. $\varepsilon^{\prime}$ and $\varepsilon^{\prime \prime}$ are the real and imaginary parts of the dielectric constant $\varepsilon$. If the imaginary part of the dielectric constant is close to zero, then the refractive index $n$ is equal to $\sqrt{\varepsilon}$. The diffraction grating period, width ratio, and depth are $380 \mathrm{~nm}, 0.5 \mathrm{~nm}$, and $35 \mathrm{~nm}$, respectively. At the same time, the thicknesses of the metal (gold) and the waveguide layer $\mathrm{Ta}_{2} \mathrm{O}_{5}$ are $40 \mathrm{~nm}$ and $285 \mathrm{~nm}$, respectively.

The change in the momentum of SPs includes a conjugated body interaction and a slight refractive index change on the surface of the metal film buffer. Therefore, the light propagation in the optical path in the diffraction grating waveguide structure does not occur. A shift in the reflective resonant wavelength occurs instead. We can observe the dynamics of the molecular interactions in situ at the sensing layer by investigating the change in the reflection of the resonance wavelength with time. Figure 2(b) shows whether the sensor in the pure water reflection spectrum simulation diagram contains molecules $\left(n_{a}=1.46\right)$ with a 10-nm-thickness layer. The resonant wavelength caused by the movement of the molecular layer is $12.4 \mathrm{~nm}$. The sensitivity of the subwavelength grating waveguide sensor determined by the current research team is approximately 10 times higher than that developed by a previous study.

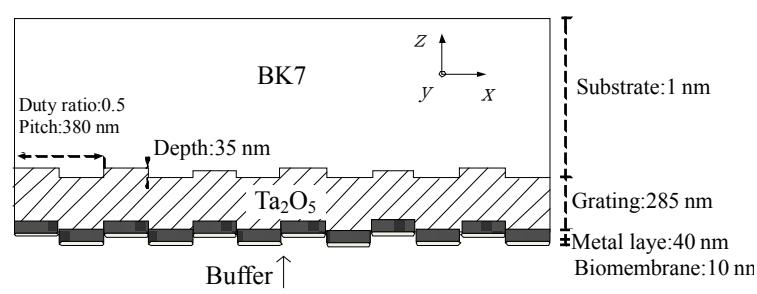

(a)

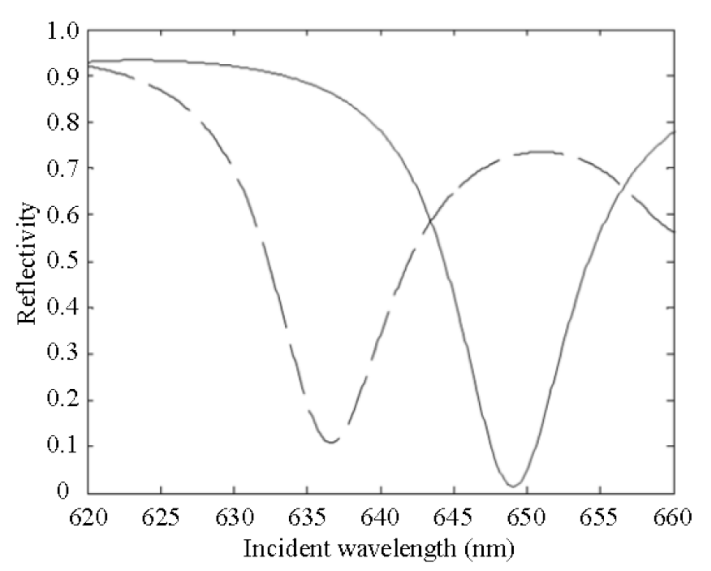

(b)

Fig. 2 Waveguide schematic: (a) waveguide grating structure of the SPR sensor and (b) diffraction efficiency of the plasma at the sensor reflector buffer.

\section{Experiment}

\subsection{Finite-difference time-domain (FDTD) analysis}

Figure 3 shows that the incident light wavelength is $637 \mathrm{~nm}$, which is obtained by using the magnetic FDTD results in the distribution in the $y$ axis. $z=0$ 
defines the interface of the molecular layer and the metal film. We observed that, as a result of surface plasma excitation at the interface, the magnetic field was significantly enhanced. Moreover, in consideration of the dielectric layer waveguide mode coupling, the wavelength of the reflection spectrum bandwidth decreased. In the spectrum, the waveguide mode was produced more clearly, significantly improving the accuracy of the measurement. At the same time, compared with the diffraction grating waveguide sensor, the plasma effect significantly increased the sensitivity of the sensor.

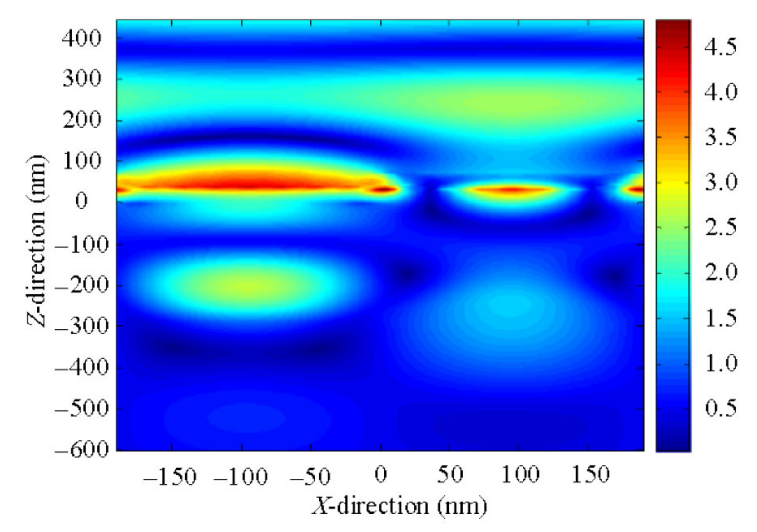

Fig. 3 FDTD results in the distribution in the $y$ axis.

\subsection{Experiment and result analysis}

Figure 4 shows the SPR sensor with a subwavelength grating reflection spectrum. When the incident white light was the TE mode, the absorption displacement was not observed in the reflection spectrum. When the incident white light was the TM mode, two absorption displacements were observed in the reflection spectrum. As the incident light was a focused beam rather than a parallel beam, the absorption displacement width was wider than that in the simulation results. In the simulation, the reflection spectrum depended on the incident angle and wavelength. In the measurement, the incident white light was gathered in the sensing area. As a result, the reflection spectrum was a combination of different diffractions. Thus, in the experimental results, the reflection spectrum became wider.

Figure 5 shows the change in the resonance wavelength, which is the basis of designing sulfur sensors in the process of dynamic response. When the reflection resonant wavelength changed to a constant, $0.5-\mu \mathrm{m}$-thickness protein was injected into the reaction chamber. After $64 \mathrm{~min}$, the 10-mmthickness Tris- $\mathrm{HCl}$ buffer was used to flush the reaction chamber. The protein on the surface of the sensor improved absorption, resulting in a resonant wavelength of $0.3 \mathrm{~nm}$.

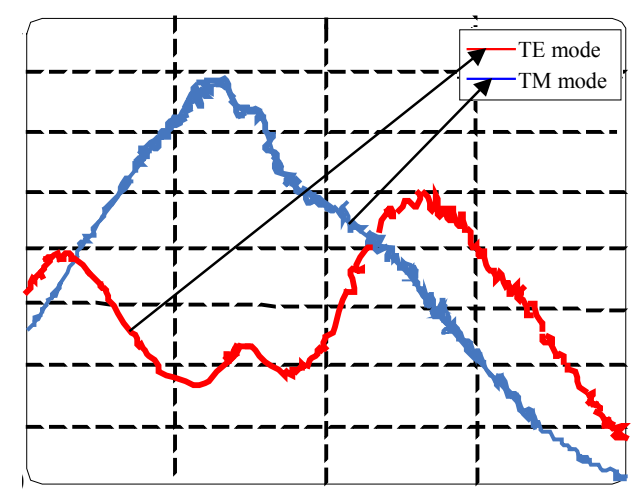

Fig. 4 SPR reflection spectrum of the sensor.

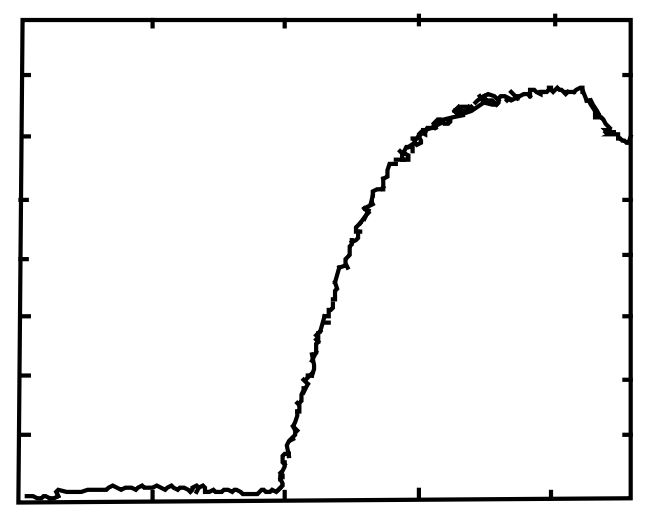

Fig. 5 Dynamic response of the sensor.

After $97 \mathrm{~min}$, antibodies were injected into the reaction chamber. The reaction lasted for approximately $2 \mathrm{~h}$. The maximum corresponding value of the resonant wavelength shift was $6.8 \mathrm{~nm}$. After $212 \mathrm{~min}$, the Tris- $\mathrm{HCl}$ buffer was injected into the reaction chamber to flush the nonspecific 
adhesive, resulting in a decrease in the resonant wavelength shift to $5.7 \mathrm{~nm}$. Using a homemade angle adjustment SPR sensor system to study the antibody $-\mathrm{G}$ protein interaction, we determined that it could lead to an SPR angle deviation of approximately $0.5^{\circ}$. As a result, the sensor has the ability to detect changes, with a detection sensitivity of $10^{-6}$ RIU based on the comparison.

\section{Summary}

The designed subwavelength grating waveguide SPR sensor provides a possible and direct optical sensing platform for real-time analysis of molecular interaction. The simulation results showed that the electrolyte layer coupling waveguide mode caused more dramatic shifts in the reflection spectrum of the SPR sensor, resulting in a higher measureable resolution. Compared with the diffraction grating sensor with no metal layer, the surface plasma model significantly improved the sensitivity of the SPR sensor. In particular, the SPR sensor had a detection sensitivity of $10^{-6}$ RIU. Therefore, the designed SPR sensor provides a powerful tool for real-time analysis of molecular interactions without the need for external markers or complex optical devices.

\section{Acknowledgment}

This work was supported by the National Natural Science Foundation of China (No. 61061006) and Guangxi Normal University Doctor Scientific Research Foundation.

Open Access This article is distributed under the terms of the Creative Commons Attribution License which permits any use, distribution, and reproduction in any medium, provided the original author(s) and source are credited.

\section{References}

[1] P. Berini, "Plasmon-polariton waves guided by thin lossy metal films of finite width: bound modes of symmetric structures," Physical Review B, 2000, 611(15): 10484-10503.
[2] T. Okamoto, F. H'Dhili, and S. Kawata, "Towards plasmonic band gap laser," Applied Physics Letters, 2004, 85(18): 3968-3970.

[3] G. Winter, S. Wedge, and W. L. Barnes, "Can lasing at visible wavelength be achieved using the low-loss long-range surface plasmon-polariton mode?" New Journal of Physics, 2006, 8(125): $1-14$.

[4] M. Z. Alam, J. Meier, J. S. Aitchison, and M. Mojahedi, "Gain assisted surface plasmon polariton in quantum well structures," Optics Express, 2007, 15(1): 176-182.

[5] M. Ambati, D. A. Genov, R. F. Oulton, and X. Zhang, "Active plasmonics: surface plasmon interaction with optical emitters," IEEE Journal of Selected Topics in Quantum Electronics, 2008, 14(6): 1395-1403.

[6] A. Kovyakov, A. R. Zakharian, K. M. Gundu, and S. A. Darmanyan, "Giant optical resonances due to gain-assisted Bloch surface plasmon," Applied Physics Letters, 2009, 94(15): 151111-1-151111-3.

[7] P. Berini, "Long-range surface plasmon polaritons," Advances in Optics and Photonics, 2009, 1(3): 484-588.

[8] I. D. Leon and P. Berini, "Modeling surface plasmonpolariton gain in planar metallic structures," Optics Express, 2009, 17(22): 20191-20202.

[9] I. D. Leon and P. Berini, "Amplification of long-range surface plasmons by a dipolar gain medium," Nature Photonics, 2010, 4(6): 382-387.

[10] M. C. Gather, K. Meerholz, N. Danz, and K. Leosson, "Net optical gain in a plasmonic waveguide embedded in a fluorescent polymer," Nature Photonics, 2010, 4(7): 457-461.

[11] F. H'Dhili, T. Okamoto, J. Simonen, and S. Kawata, "Improving the emission efficiency of periodic plasmonic structures for lasing applications," Optics Communications, 2011, 284(2): 561-566.

[12] Y. Chen and L. Guo, "High $Q$ long-range surface plasmon polariton modes in sub-wavelength metallic microdisk cavity," Plasmonics, 2011, 6(1): 183-188.

[13] I. D. Leon and P. Berini, "Spontaneous emission in long-range surface plasmonpolariton amplifiers," Physical Review B, 2011, 83(8): 081414(R).

[14] I. D. Leon and P. Berini, "Measuring gain and noise in active long-range surface plasmon-polariton waveguides," Review of Scientific Instruments, 2011, 82(3): 033107.

[15] R. A. Flynn, C. S. Kim, I. Vurgaftman, M. Kim, J. R. Meyer, A. J. Mäkinen, et al., "A room-temperature semiconductor spaser operating near $1.5 \mu \mathrm{m}$," Optics Express, 2011, 19(9): 8954-8961.

[16] D. F. P. Pile, T. Ogawa, D. K. Gramotnev, Y. Matsuzaki, K. C. Vernon, K. Yamaguchi, et al., "Two-dimensionally localized modes of a nanoscale gap plasmon waveguide," Applied Physics Letters, 
2005, 87(26): 261114.

[17] J. A. Dionne, L. A. Sweatlock, H. A. Atwater, and A. Polman, "Plasmon slot waveguides: towards chip-scale propagation with subwavelength-scale localization," Physical Review B, 2006, 73(3): 035407.

[18] S. A. Maier, "Gain-assisted propagation of electromagnetic energy in subwavelength surface plasmon polariton gap waveguides," Optics Communications, 2006, 258(2): 295-299.

[19] Z. Yu, G. Veronis, S. Fan, and M. L. Brongersma, "Gain-induced switching in metal-dielectric-metal plasmonic waveguides," Applied Physics Letters, 2008, 92(4): 041117-1-041117-3. 